\title{
ON THE CIRCULAR RELATION OF MÖBIUS.
}

[From the Proceedings of the London Mathematical Society, vol. vill. (1876-1877), pp. 220-225. Read April 12, 1877.]

IN representing a given imaginary or complex quantity $u,=x+i y$, by means of the point whose coordinates are $x, y$, we assume in the first instance that $x, y$ are real,-but in the results this restriction may be abandoned-for instance, if the imaginary quantities $u, u^{\prime}, c$ are connected by the equation $u^{2}+u^{\prime 2}=c^{2}$; then, writing $u=x+i y$, $u^{\prime}=x^{\prime}+i y^{\prime}, \quad c=a+b i$, we have $x^{2}-y^{2}+x^{\prime 2}-y^{\prime 2}=a^{2}-b^{2}, \quad x y+x^{\prime} y^{\prime}=a b$, equations connecting the points $U, U^{\prime}, C$ which serve to represent the quantities $u, u^{\prime}, c$, and which (regarding $C$ as a fixed point) establish a correspondence between the two variable points $U, U^{\prime}$ : any given value $u,=x+i y$, is reprcsented by the point $U$, and corresponding hereto we have (in the present case) two points $U^{\prime}$, viz. these are the real intersections of the curves $x^{\prime 2}-y^{\prime 2}=a^{2}-b^{2}-\left(x^{2}-y^{2}\right), \quad x^{\prime} y^{\prime}=a b-x y$, and then the coordinates $x^{\prime}, y^{\prime}$ of either of these give the value $x^{\prime}+i y^{\prime}$ of $u^{\prime}$.

But, the two curves once arrived at, we may for other purposes be concerned with their intersections as well imaginary as real; or, still more generally, all the quantities entering into the two equations may be regarded as imaginary.

Theoretically we seem to require two imaginary roots of unity, incommensurable and convertible, viz. taking these to be $i, I$, then $i^{2}=-1, I^{2}=-1, i I=I i$, but without any relation between $i, I$ : thus, in what precedes, writing $I$ instead of $i$, viz. $u, u^{\prime}, c=x+I y, x^{\prime}+I y^{\prime}, a+b I$, here each of the quantities $x, y, x^{\prime}, y^{\prime}, a, b$ can be $a b$ initio an imaginary quantity of the form $\lambda+\mu i$. But, conforming to the ordinary practice, I use $i$ only, writing for instance $u=x+i y$, without any express statement that $x, y$ are real; on the understanding that any equation containing such quantities, and therefore ultimately of the form $P+i Q=0$, denotes the two equations $P=0, Q=0$ (or, what is the same thing, that we have not only the original equation, but, in addition to it, the like equation with each such quantity $x+i y$ replaced by the con- 
jugate quantity $x-i y)$ : and the further understanding that, in the pair of equations, each of the quantities $x, y$, \&c. entering therein may itself be considered as an imaginary quantity of the form $\lambda+\mu i$.

The foregoing explanation is required, for otherwise it would appear as if the circular relation of Möbius* about to be explained was of necessity a relation between real points: I hold that this is not the case. But in all that follows I do, in fact, consider primarily the case of real points; and indeed the occasion does not arise for any explicit consideration of the case of imaginary points.

The circular relation is as follows. If in the first instance we have four points $U, A, B, C$ on a line, and $u, a, b, c$ their distances from any fixed point on that line; and again, $U^{\prime}, A^{\prime}, B^{\prime}, C^{\prime}$ four other points on a line (the same or a different line), and $u^{\prime}, a^{\prime}, b^{\prime}, c^{\prime}$ their distances from any fixed point on that line; then the same equation between $u, a, b, c, u^{\prime}, a^{\prime}, b^{\prime}, c^{\prime}$ which expresses the homographic relation between the two ranges of points $U, A, B, C$ and $U^{\prime}, A^{\prime}, B^{\prime}, C^{\prime}$, expresses, when differently interpreted, the circular relation between the four points $U, A, B, C$ in a plane, and the four other points $U^{\prime}, A^{\prime}, B^{\prime}, C^{\prime}$ in the same or a different planeviz. for the new interpretation, $u$ is used as denoting $x+i y$, the linear function of the coordinates $x, y$ of the point $U$, and the like as regards the remaining quantities $a, b, c, u^{\prime}, a^{\prime}, b^{\prime}, c^{\prime}$.

As in the homographic theory (but of course without the condition of being in a line), we have $A, A^{\prime} ; B, B^{\prime} ; C, C^{\prime}$ given pairs of corresponding points: the equation now represents two equations; and these, when either of the points $U, U^{\prime}$ is given, determine the corresponding point $U^{\prime}$ or $U$.

The homographic relation may be written in the forms

$$
\begin{array}{llll}
1, & u, & u^{\prime}, & u u^{\prime} \\
1, & a, & a^{\prime}, & a a^{\prime} \\
1, & b, & b^{\prime}, & b b^{\prime} \\
1, & c, & c^{\prime}, & c c^{\prime}
\end{array} \mid=0,
$$

$$
u-a \cdot b-c: u-b \cdot c-a: u-c \cdot a-b=u^{\prime}-a^{\prime} \cdot b^{\prime}-c^{\prime}: u^{\prime}-b^{\prime} \cdot c^{\prime}-a^{\prime}: u^{\prime}-c^{\prime} \cdot a^{\prime}-b^{\prime},
$$

viz. these are forms of one and the same equation: and it may be added that, if $\omega$ in the first system corresponds to $\infty$ in the second system, and $\omega^{\prime}$ in the second system to $\infty$ in the first system (of course $\omega, \omega^{\prime}$ are not corresponding values in the two systems respectively); so that

$$
\begin{aligned}
& 1, \omega=0 \text {, } \\
& 1, \quad a, \quad a^{\prime}, \quad a a^{\prime} \\
& 1, \quad b, \quad b^{\prime}, \quad b b^{\prime} \\
& 1, \quad c, \quad c^{\prime}, \quad c c^{\prime} \\
& \text { 1, } \omega^{\prime}=0 \text {, } \\
& \text { 1, } a, a^{\prime}, a a^{\prime} \\
& 1, \quad b, \quad b^{\prime}, b b^{\prime} \\
& 1, \quad c, \quad c^{\prime}, \quad c c^{\prime} \\
& \omega-a \cdot b-c: \omega-b \cdot c-a: \omega-c \cdot a-b=b^{\prime}-c^{\prime}: c^{\prime}-a^{\prime}: a^{\prime}-b^{\prime} \text {; } \\
& b-c: \quad c-a: \quad a-b=\omega^{\prime}-a^{\prime} \cdot b^{\prime}-c^{\prime}: \omega^{\prime}-b^{\prime} \cdot c^{\prime}-a^{\prime}: \omega^{\prime}-c^{\prime} \cdot a^{\prime}-b^{\prime} ; \\
& \text { * [Möbius, Ges. Werke; t. II., pp. 243-314, and elsewhere.] }
\end{aligned}
$$


whence also, if

$$
\Lambda=\left|\begin{array}{lll}
1, & 1, & 1 \\
a, & b, & c \\
a^{\prime}, & b^{\prime}, & c^{\prime}
\end{array}\right|,=b c^{\prime}-b^{\prime} c+c a^{\prime}-c^{\prime} a+a b^{\prime}-a^{\prime} b
$$

then

$$
\begin{array}{ll}
-\Lambda \cdot \omega-a=b-c \cdot c^{\prime}-a^{\prime} \cdot a^{\prime}-b^{\prime}, & \Lambda \cdot \omega^{\prime}-a^{\prime}=b^{\prime}-c^{\prime} \cdot c-a \cdot a-b, \\
-\Lambda . \omega-b=c-a \cdot a^{\prime}-b^{\prime} \cdot b^{\prime}-c^{\prime}, & \Lambda \cdot \omega^{\prime}-b^{\prime}=c^{\prime}-a^{\prime} \cdot a-b \cdot b-c, \\
-\Lambda . \omega-c=a-b . b^{\prime}-c^{\prime} \cdot c^{\prime}-a^{\prime}, & \Lambda \cdot \omega^{\prime}-c^{\prime}=a^{\prime}-b^{\prime} \cdot b-c \cdot c-a .
\end{array}
$$

Then, $\omega, \omega^{\prime}$ being thus determined, we have

$$
\begin{aligned}
& \omega-a \cdot \omega^{\prime}-a^{\prime}=\omega-b \cdot \omega^{\prime}-b^{\prime}=\omega-c \cdot \omega^{\prime}-c^{\prime}=\omega-u \cdot \omega^{\prime}-u^{\prime} \\
&=-\frac{b-c \cdot c-a \cdot a-b \cdot b^{\prime}-c^{\prime} \cdot c^{\prime}-a^{\prime} \cdot a^{\prime}-b^{\prime}}{\Lambda^{2}}(=\Delta \text { suppose }),
\end{aligned}
$$

viz we have $\omega-u \cdot \omega^{\prime}-u^{\prime}=$ a given value; which is the most simple form of the relation between $u, u^{\prime}$.

Interpreting everything in the first instance in regard to the homographic ranges, the equations show that there is in the first range a point $O$, and in the second range a point $O^{\prime}$, such that $O A$, \&c. denoting distances, we have

$$
O A \cdot O^{\prime} A^{\prime}=O B \cdot O^{\prime} B^{\prime}=O C \cdot O^{\prime} C^{\prime}=O U \cdot O^{\prime} U^{\prime}(=\Delta) ;
$$

or, what is the same thing, if in the line of the first range we construct $A_{1}, B_{1}, C_{1}, U_{1}$ by the formulæ

$$
O A \cdot O A_{1}=O B . O B_{1}=O C \cdot O C_{1}=O U \cdot O U_{1}=\Delta,
$$

that is, invert the first range in regard to the centre $O$ and squared radius $\Delta$, then we have a range $O, A_{1}, B_{1}, C_{1}, U_{1}$ equal to the range $O^{\prime}, A^{\prime}, B^{\prime}, C^{\prime}, U^{\prime}$; viz. the distances of corresponding points are equal in the two cases: or say a range $O, A_{1}, B_{1}, C_{1}, U_{1}$ imposable upon $O^{\prime}, A^{\prime}, B^{\prime}, C^{\prime}, U^{\prime}$.

The like result holds for the circular relation, but the interpretation must be explained more in detail. And, first, it is to be remarked that $O$ in the first figure is the point corresponding to any point whatever at infinity in the second figure; viz. writing $u^{\prime}=\xi^{\prime}+i \eta^{\prime},=\infty$, then, whatever value we give to the ratio of the two infinite quantities $\xi^{\prime}, \eta^{\prime}$, we obtain the same complex value of $\omega$, that is, the same coordinates for the point $O$. And, similarly, $O^{\prime}$ in the second figure is the point corresponding to any point whatever at infinity in the first figure.

To determine $O$, we have the equation

$$
\frac{\omega-a}{\omega-b}=\frac{b^{\prime}-c^{\prime}}{c^{\prime}-a^{\prime}} \cdot \frac{c-a}{a-b} .
$$

Any such equation gives at once the geometrical construction, viz. $\omega-a=O A e^{i O A x}$, where $O A$ is the distance of the points $O, A$ regarded as positive, and $O A x$ is the 
inclination of the line $O A$ regarded as drawn from $A$ to $O$ to the line $A x$, such angle being measured in the sense $A x$ to $A y$; where $A x, A y$ are the lines drawn from $A$ in the senses $x$ positive and $y$ positive respectively: and so in other cases.

The equation is therefore equivalent to the two equations

$$
\frac{O A}{O B}=\frac{B^{\prime} C^{\prime}}{C^{\prime} A^{\prime}} \cdot \frac{C A}{A B}
$$

and

$$
\angle O A x-\angle O B x=\angle B^{\prime} C^{\prime} x-\angle C^{\prime} A^{\prime} x+\angle C A x-\angle A B x .
$$

The former of these expresses that $O$ is in a certain circle which, having its centre on the line $A B$, cuts $A B$ and $A B$ produced in the one or the other sense; the latter that it is in the segment described on a determinate side of $A B$ and containing a given angle: hence $O$, as the intersection of the segment with the first-mentioned circle, is a uniquely determined point. Similarly $O^{\prime}$ is a uniquely determined point.

It is not obvious how to construct $\Lambda$, from its original value as given above (but, $\omega$ being known, we can without difficulty construct it from the value

$$
\left.-\Lambda \cdot \omega-a=b-c \cdot c^{\prime}-a^{\prime} \cdot a^{\prime}-b^{\prime}\right),
$$

nor consequently $\Delta$ from its expression in terms of $\Lambda$ : but, $\omega$ and $\omega^{\prime}$ being known, we can construct $\Delta$ from the expression $\omega-a \cdot \omega^{\prime}-a^{\prime}=\Delta$; supposing it thus constructed, $=k e^{i \theta}$ suppose, then if, with centre $O$ and squared radius $k$, we invert the first figure, thereby obtaining the points $A_{1}, B_{1}, C_{1}, U_{1}$ such that

$$
O A \cdot O A_{1}=O B \cdot O B_{1}=O C \cdot O C_{1}=O U \cdot O U_{1}=k,
$$

(the points $A_{1}, B_{1}, C_{1}, U_{1}$ being on the lines $O A, O B, O C, O U$ respectively,) then the equations

$$
\omega-a \cdot \omega^{\prime}-a^{\prime}=\omega-b \cdot \omega^{\prime}-b^{\prime}=\omega-c \cdot \omega^{\prime}-c^{\prime}=\omega-u \cdot \omega^{\prime}-u^{\prime}=k e^{i \theta} .
$$

give

that is,

$$
\omega-a . \omega^{\prime}-a^{\prime}=O A . O A_{1} e^{i \theta}
$$

and

$$
O A \cdot O^{\prime} A^{\prime}=O A . O A_{1} \text {, or, simply, } O^{\prime} A^{\prime}=O A_{1}
$$

$$
\angle A O x+\angle A O^{\prime} x^{\prime}=\theta,
$$

or, what is the same thing,

$$
\angle A_{1} O x+\angle A^{\prime} O x^{\prime}=\theta
$$

and so for the other letters, viz. we have

$$
O^{\prime} A^{\prime}, O^{\prime} B^{\prime}, O^{\prime} C^{\prime}, O^{\prime} U^{\prime}=O A_{1}, O B_{1}, O C_{1}, O U_{1},
$$

respectively; and further

$$
\angle \text { 's } A_{1} O x, B_{1} O x, C_{1} O x, U_{1} O x=\theta-A^{\prime} O^{\prime} x^{\prime}, \theta-B^{\prime} O^{\prime} x^{\prime}, \theta-C^{\prime} O^{\prime} x^{\prime}, \theta-U^{\prime} O^{\prime} x^{\prime} \text {, }
$$


respectively; viz. the system of points $O, A_{1}, B_{1}, C_{1}, U_{1}$ is equal to the system $O^{\prime}, A^{\prime}, B^{\prime}, C^{\prime}, U^{\prime}$, that is, the distances of corresponding points and magnitudes of corresponding angles are severally equal-but the angles $A_{1} O x$ and $A^{\prime} O^{\prime} x^{\prime}$, \&c. are in opposite senses, as appears by the just mentioned equations $A_{1} O x=\theta-A^{\prime} O^{\prime} x^{\prime}$, \&c.; that is, the two figures are symmetrically equal: but the one of them is not, except by a turning over of its plane, imposable upon the other.

The conclusion is, the two figures $A, B, C, U$ and $A^{\prime}, B^{\prime}, C^{\prime}, U^{\prime}$ are each of them equal by symmetry, but not superimposably, to a figure which is the inverse of the other of them: viz. there exists in the first figure a point $O$, and in the second figure a point $O^{\prime}$, such that, inverting say the first figure, with centre $O$ and a squared radius of determinate magnitude, we obtain the points $A_{1}, B_{1}, C_{1}, U_{1}$, forming with $O$ a figure equal by symmetry, but not superimposably, to the second figure $A^{\prime}, B^{\prime}, C^{\prime}, U^{\prime}, O^{\prime}$. Hence also to any line in the first figure corresponds in the second figure a circle through $O^{\prime}$, and to any line in the second figure there corresponds in the first figure a circle through $O$; or, more generally, to any circle in either figure there sorresponds a circle in the other figure.

There is a particular case of peculiar interest, viz. writing for greater convenience $d, d^{\prime}$ as corresponding values in place of $u, u^{\prime}$, the system $a, b, c, d$ corresponds homographically to itself in three different ways; that is, we may have

$$
\left(a^{\prime}, b^{\prime}, c^{\prime}, d^{\prime}\right)=(b, a, d, c),(c, d, a, b) \text { or }(d, c, b, a) .
$$

To fix the ideas, attending to the first. case, we have thus the range of points $(A, B, C, D)$ corresponding homographically to $(B, A, D, C)$, viz. here $\omega^{\prime}=\omega$, and $\omega-a \cdot \omega-b=\omega-c \cdot \omega-d$, that is, the corresponding points $U, U^{\prime}$ belong to the involution where $A$ and $B$ and also $C$ and $D$ are corresponding points. The like theory applies to the circular transformation: viz. the points $(A, B, C, D)$ may correspond to $(B, A, D, C)$, viz. there exists a point $O$ (or say $C_{1}$ ) and squared radius $k_{1}$, such that, inverting the figure and marking the inverse points of $A, B, C, D$ as $B_{1}, A_{1}, D_{1}, C_{1}$ respectively, the new figure $O_{1}, A_{1}, B_{1}, C_{1}, D_{1}$ is equal by symmetry, but not superimposably, to the original figure $O A B C D$. The equation $\omega_{1}-a \cdot \omega_{1}-b=\omega_{1}-c \cdot \omega_{1}-d$ gives the geometrical definition of the point $O_{1}$, viz. this is a point such that $O_{1} A . O_{1} B=O_{1} C \cdot O_{1} D$ and further that $A B$ and $C D$ subtend at $O_{1}$ equal angles: we have $\omega_{1}=\frac{a b-c d}{a+b-c-d}$, giving for $\omega_{1}-a, \omega_{1}-b, \omega_{1}-c, \omega_{1}-d$ convenient expressions the first of which is $\omega_{1}-a=\frac{c-a \cdot d-a}{c+d-a-b}$. We hence obtain a convenient construction for $O$, viz. taking $M$ for the middle point of $A B$ and $N$ for the middle point of $C D$, and drawing from $A$ in the sense $M$ to $N$ a line $A P,=2 M N$, then this equation may be written $\omega_{1}-a=\frac{c-a \cdot d-a}{p-a}$ ( $p$ the function $x+i y$ which belongs to the point $P) ;$ thence $O_{1} A=\frac{C A \cdot D A}{P A}$ and

$$
\angle O_{1} A x=\angle C A x+\angle D A x-\angle P A x,
$$

conditions which determine uniquely the position of $O_{1}$. 
We may have $(A, B, C, D)$ corresponding to $(C, D, A, B)$ and $(D, C, B, A)$, the inversions for these depending on the points $O_{2}$ and $O_{3}$ respectively: I annex a figure showing the three inversions of the same four points $A, B, C, D$.

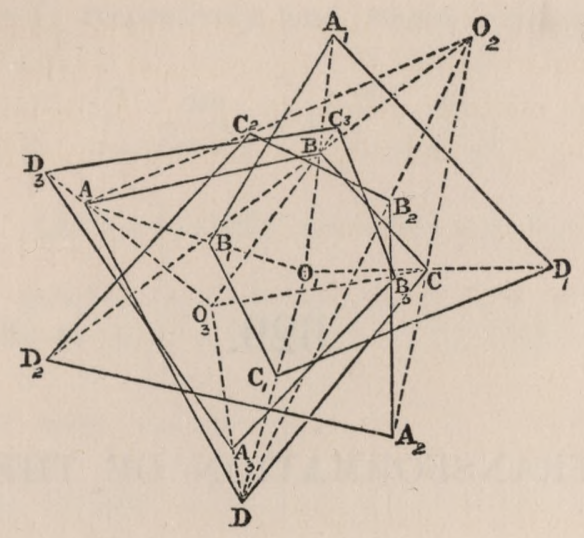

